\title{
Late palliative care of patients with lung cancer may have a limited effect on quality of life - a pilot observational study
}

Andrzej Nowicki ${ }^{1}$, Paulina Farbicka ${ }^{1}$, Małgorzata Krajnik ${ }^{2}$

${ }^{1}$ Department of Oncology Nursing, Collegium Medicum in Bydgoszcz, Nicolaus Copernicus University in Torun, Poland

${ }^{2}$ Department and Palliative Care Unit, Collegium Medicum in Bydgoszcz, Nicolaus Copernicus University in Torun, Poland

Submitted: 18 March 2016

Accepted: 10 April 2016

Arch Med Sci Civil Dis 2016; 1: e1-e9

DOI: 10.5114/amscd.2016.59584

Copyright $\odot 2016$ Termedia \& Banach

\author{
Corresponding author: \\ Andrzej Nowicki \\ Department of Oncology \\ Nursing \\ Collegium Medicum \\ Nicolaus Copernicus \\ University \\ 3 Techników St \\ 85-801 Bydgoszcz, Poland \\ Phone: +48602 383210 \\ E-mail: anow1_xl@wp.pl
}

\begin{abstract}
Introduction: The aim of the study was to assess the point of life and illness at which patients began to receive palliative care and in what areas of their quality of life effective alleviation of their suffering is possible.

Material and methods: The study included 63 patients aged 33-91 years with lung cancer treated in palliative medicine centers in Bydgoszcz in 2012-2013. The assessment of quality of life was performed every three weeks, using the QLQ-C30 and QLQ-LC13 questionnaires.

Results: The median time from diagnosis to the start of palliative care was 10 months, and the median palliative care duration for the whole group was 4 weeks. Patients with a survival time less than 6 weeks $(n=42)$ experienced worse symptoms and limitations in their functioning during the whole period of care until their death compared with those who survived a little longer (6-8 weeks; $n=21)$. Fatigue, constipation and dyspnea were among the worst symptoms; the latter intensified in the last period before death. The quality of life assessed by patients continuously deteriorated, and in the last days/ weeks before death it was defined as "very bad" by more than $2 / 3$ of patients. Conclusions: Late provision of patients with palliative care can lead to its limited effectiveness in improving the quality of life, which is dependent on symptoms that are experienced by patients and ability to function in the physical, emotional, cognitive, social and societal spheres.
\end{abstract}

Key words: lung cancer, quality of life, palliative care, early integration.

\section{Introduction}

Lung cancer accounts for $12 \%$ of all cancers, and it is characterized by the highest annual mortality rate among cancers in men and women worldwide [1]. In Poland, in 2010 lung cancer was the most commonly diagnosed cancer in men and for this reason was the most common cause of death in both men and women [2]. Tumor biology itself, late diagnosis, advanced age, presence of comorbidities as well as limitations in the possibilities of anticancer treatment resulting from mentioned factors result in a poor prognosis. Moreover, patients with lung cancer have the highest number of symptoms among all cancer patients [3].

Suffering due to the great discomfort caused by many symptoms can be multiplied by the prospect of the inevitability of death and ideas of 
what it will be like $[4,5]$. It is no wonder that studies to evaluate the effects of early implementation of palliative care in the full model of integrating the activities in the field of palliative medicine and oncology have been started [6]. It turned out that introduction of early palliative care in conjunction with the standard oncological practice through holistic alleviation of patient suffering can not only improve the quality of life but also improve survival of patients [7-10].

The opinion of the American Society of Clinical Oncology (ASCO), which was published in 2012, indicates the need to ensure introduction of simultaneous cancer treatment and palliative care as early as possible to all patients with advanced cancer regardless of cancer stage in case of the presence of many symptoms or other problems [11]. Various oncology centers in the world are trying to develop models for the early integration of palliative care with oncology [12-14]. There is a well-developed network of specialized palliative care services for cancer patients in Poland. However, there are no data on the practice for providing patients with advanced lung cancer with palliative care. There is only an individual analysis of the impact of palliative care on the quality of life in these patients [15].

The present study aims to assess at which point of a patient's life and illness patients with advanced lung cancer begin to receive palliative care in Poland and in what areas of the quality of life the patient's suffering can be alleviated effectively and in what areas it cannot be alleviated.

\section{Material and methods}

The study included patients with lung cancer treated in palliative medicine centers in Bydgoszcz in 2013-2014, being under the care of palliative medicine clinics, home palliative care teams or staying in a hospice inpatient unit. Assessment of the quality of life was carried out every three weeks, at least twice. The first assessment could be made at least 7 days after the start of palliative care. Patients with other cancers occurring with the presence of CNS metastases were excluded from the study.

The QLQ-C30 questionnaire to assess the quality of life in patients treated for cancer and the QLQ-LC13 module designed for patients with lung cancer were used in the study. Each question (with the exception of questions 29 and 30 in the QLQ-C30) required marking an answer on a 4-point scale characterizing the severity of the symptoms associated with the level of quality of life. A method for calculation of the quality of life of a given patient consisting of normalization (bringing all ratings to a $0-1$ scale) and calculation of the final result as the average score of all answers was proposed. The quality of life of each patient was assessed on a scale from 0 (worst quality of life) to 1 - the best quality of life (excellent). After the linear transformation, answers in the questionnaires received the following values: 'not at all' -1 , 'a little' -0.67 , significantly' -0.33 and 'very' -0 .

The study was approved by the local Research Ethics Committee of Nicolaus Copernicus University, Collegium Medicum in Bydgoszcz, Poland, and all subjects gave written informed consent.

\section{Statistical analysis}

Pearson correlation coefficients were calculated in order to detect possible relationships between measurable characteristics. A parametric test was used for two coefficients of structure and a nonparametric test of independence, the $\chi^{2}$ test, in order to detect correlations between non-measurable and qualitative characteristics. In case of a small size of one of the classes, the $\chi^{2}$ test with Yates correction was used. Student's $t$ test was also used for dependent groups and verification of the hypothesis of the significance of correlation coefficients. In order to verify the hypothesis of the significance of reducing the level of quality of life in various spheres of functioning, variations in the quality of life in a given sphere were calculated for each patient. Subsequently, the average value of change was calculated and the null hypothesis was verified using Student's $t$ test for dependent groups. The significance level $p=0.05$ was adopted. Additionally, an ANOVA was performed to determine differences in the quality of life in its particular spheres between successive tests. The Wilcoxon matched pairs test was used to evaluate differences between obtained results.

\section{Results}

\section{Characteristics of patients undergoing palliative care}

In total 228 patients were enrolled in the study. Seventy-six persons did not complete questionnaires because of difficulties with logical contact, 4 questionnaires were filled only once, and 85 persons refused to participate in the study. Finally, the study included 63 persons (21 women and 42 men), who completed the questionnaire at least twice. The median age of the study group was 66 years (range: 33-91). They were patients who were initially treated at home $(n=59)$ or in a palliative care clinic $(n=4)$. At the end of the first month of palliative care (study 2 ) its profile changed: patients were treated at home $(n=55)$, or they were admitted to a hospice $(n=8)$. In 63 patients assessment of the quality of life was made twice (the first time within 7 days after the start of palliative care, the second time 3 weeks 
after the first assessment), while the third assessment was made in 21 patients (6 weeks after the first assessment). Overall, 147 questionnaires were completed.

The median time from diagnosis to the start of palliative care was 10 months. In 35 patients the time from diagnosis was up to 12 months, in 23 patients it was 24 months, and in 5 patients it was above 24 months.

Of the 63 patients involved in the study, 42 died between the second and third assessment, i.e. between the third and sixth week after the start of palliative care. Among 21 patients who passed the third assessment of the quality of life 6 weeks after the first assessment, 3 patients died at 43 days, 5 at 44 days, 9 at 46 days, 3 at 47 days and 1 at 51 days after the start of palli- ative care. The median time of palliative care for the entire group was four weeks.

Changes of the quality of life depended on existing symptoms and physical, emotional, social, societal and cognitive functioning (Tables I-III)

At the moment of the start of palliative care (study $1, n=63$ ), $81 \%$ of patients had serious or very serious problems with going out for a short walk, $82 \%$ with performing strenuous activities and $71.4 \%$ reported the need to stay in bed or sit in a chair during the day. $47.6 \%$ of patients needed help with eating, dressing, washing or using a toilet very or greatly. Significant or very significant limitations in family and social life occurred in $23.8 \%$ and $58.7 \%$, respectively, while limitations

Table I. Quality of life associated with symptoms assessed in $1^{\text {st }}$ and $2^{\text {nd }}$, and $1^{\text {st }}$ and $3^{\text {rd }}$ study

\begin{tabular}{|c|c|c|c|c|c|c|}
\hline \multirow[t]{3}{*}{ Symptoms (question) } & Study 1 & Study 2 & \multirow[t]{3}{*}{$P$-value } & Study 1 & Study 3 & \multirow[t]{3}{*}{$P$-value } \\
\hline & \multicolumn{2}{|c|}{$N=63$} & & \multicolumn{2}{|c|}{$N=21$} & \\
\hline & Average \pm SD & Average \pm SD & & Average \pm SD & Average \pm SD & \\
\hline Dyspnea (8) & $0.56 \pm 0.26$ & $0.30 \pm 0.26$ & 0.0001 & $0.65 \pm 0.27$ & $0.30 \pm 0.26$ & 0.0001 \\
\hline Pain (9): & $0.48 \pm 0.24$ & $0.41 \pm 0.23$ & 0.04 & $0.37 \pm 0.26$ & $0.36 \pm 0.25$ & 0.013 \\
\hline In the chest (40) & $0.54 \pm 0.26$ & $0.45 \pm 0.25$ & 0.008 & $0.55 \pm 0.26$ & $0.35 \pm 0.22$ & 0.02 \\
\hline Arm or shoulder (41) & $0.91 \pm 0.22$ & $0.95 \pm 0.15$ & 0.019 & $0.87 \pm 0.26$ & $0.92 \pm 0.23$ & 0.33 \\
\hline In other part of the body (42) & $0.88 \pm 0.24$ & $0.89 \pm 0.28$ & 0.82 & $0.95 \pm 0.11$ & $0.94 \pm 0.17$ & 0.37 \\
\hline Difficulty sleeping (11) & $0.68 \pm 0.25$ & $0.48 \pm 0.39$ & 0.0001 & $0.79 \pm 0.17$ & $0.65 \pm 0.31$ & 0.075 \\
\hline Weakness (12) & $0.20 \pm 0.28$ & $0.14 \pm 0.27$ & 0.014 & $0.46 \pm 0.29$ & $0.08 \pm 0.15$ & 0.0001 \\
\hline Lack of appetite (13) & $0.61 \pm 0.31$ & $0.47 \pm 0.35$ & 0.0001 & $0.84 \pm 0.23$ & $0.56 \pm 0.27$ & 0.001 \\
\hline Nausea (14) & $0.93 \pm 0.14$ & $0.97 \pm 0.09$ & 0.04 & $0.89 \pm 0.16$ & $0.97 \pm 0.10$ & 0.21 \\
\hline Vomiting (15) & $0.98 \pm 0.07$ & $1.0 \pm 0.07$ & 0.083 & $0.98 \pm 0.07$ & $1.0 \pm 0.0$ & 0.33 \\
\hline Constipation (16) & $0.44 \pm 0.42$ & $0.19 \pm 0.33$ & 0.0001 & $0.76 \pm 0.34$ & $0.27 \pm 0.36$ & 0.0001 \\
\hline Diarrhea (17) & $0.97 \pm 0.09$ & $1.0 \pm 0.00$ & 0.023 & $0.95 \pm 0.12$ & $1.0 \pm 0.0$ & 0.083 \\
\hline Fatigue (18) & $0.22 \pm 0.28$ & $0.20 \pm 0.24$ & 0.505 & $0.48 \pm 0.29$ & $0.10 \pm 0.19$ & 0.0001 \\
\hline Cough (31) & $0.57 \pm 0.20$ & $0.67 \pm 0.26$ & 0.001 & $0.59 \pm 0.21$ & $0.71 \pm 0.16$ & 0.008 \\
\hline Hemoptysis (32) & $0.92 \pm 0.16$ & $1.0 \pm 0.0$ & 0.001 & $0.92 \pm 0.15$ & $1.0 \pm 0.0$ & 0.021 \\
\hline \multicolumn{7}{|l|}{ Breathlessness: } \\
\hline At rest (33) & $0.58 \pm 0.28$ & $0.32 \pm 0.33$ & 0.0001 & $0.75 \pm 0.23$ & $0.35 \pm 0.29$ & 0.0001 \\
\hline While walking (34) & $0.16 \pm 0.29$ & $0.08 \pm 0.20$ & 0.0001 & $0.43 \pm 0.37$ & $0.30 \pm 0.10$ & 0.0001 \\
\hline While walking up the stairs (35) & $0.15 \pm 0.29$ & $0.06 \pm 0.18$ & 0.0001 & $0.43 \pm 0.37$ & $0.0 \pm 0.0$ & 0.0001 \\
\hline Sore mouth or tongue (36) & $0.99 \pm 0.04$ & $1.0 \pm 0.0$ & 0.32 & $0.97 \pm 0.12$ & $1.0 \pm 0.07$ & 0.33 \\
\hline Problems with swallowing (37) & $0.95 \pm 0.13$ & $0.96 \pm 0.14$ & 0.43 & $0.97 \pm 0.15$ & $0.98 \pm 0.07$ & 0.66 \\
\hline Tingling in the hand or feet (38) & $0.93 \pm 0.15$ & $0.79 \pm 0.29$ & 0.002 & $0.90 \pm 0.15$ & $0.79 \pm 0.27$ & 0.11 \\
\hline Hair loss (39) & $0.87 \pm 0.25$ & $0.88 \pm 0.26$ & 0.37 & $0.90 \pm 0.24$ & $0.94 \pm 0.23$ & 0.16 \\
\hline
\end{tabular}


Table II. Quality of life related to areas of functioning in $1^{\text {st }}$ and $2^{\text {nd }}$, and $1^{\text {st }}$ and $3^{\text {rd }}$ study

\begin{tabular}{|c|c|c|c|c|c|c|}
\hline \multirow[t]{3}{*}{ Functioning (question) } & Study 1 & Study 2 & \multirow[t]{3}{*}{$P$-value } & Study 1 & Study 3 & \multirow[t]{3}{*}{$P$-value } \\
\hline & \multicolumn{2}{|c|}{$N=63$} & & \multicolumn{2}{|c|}{$N=21$} & \\
\hline & Average \pm SD & Average \pm SD & & Average \pm SD & Average \pm SD & \\
\hline \multicolumn{7}{|l|}{ Physical: } \\
\hline $\begin{array}{l}\text { Troubles with strenuous } \\
\text { activities (1) }\end{array}$ & $0.16 \pm 0.3$ & $0.11 \pm 0.21$ & 0.021 & $0.41 \pm 0.36$ & $0 \pm 0$ & 0.0001 \\
\hline Fatigue during long walk (2) & $0.14 \pm 0.24$ & $0.09 \pm 0.19$ & 0.011 & $0.37 \pm 0.3$ & $0 \pm 0$ & 0.0001 \\
\hline $\begin{array}{l}\text { Difficulty in walking } \\
\text { outside the house (3) }\end{array}$ & $0.21 \pm 0.31$ & $0.11 \pm 0.25$ & 0.0001 & $0.48 \pm 0.37$ & $0.03 \pm 0.1$ & 0.0001 \\
\hline $\begin{array}{l}\text { The necessity of lying in } \\
\text { bed or sitting in a chair } \\
\text { during the day (4) }\end{array}$ & $0.29 \pm 0.33$ & $0.16 \pm 0.29$ & 0.0001 & $0.59 \pm 0.31$ & $0.13 \pm 0.17$ & 0.0001 \\
\hline $\begin{array}{l}\text { Help needed with eating, } \\
\text { dressing, washing, using } \\
\text { the toilet (5) }\end{array}$ & $0.48 \pm 0.4$ & $0.27 \pm 0.41$ & 0.0001 & $0.78 \pm 0.32$ & $0.13 \pm 0.17$ & 0.0001 \\
\hline \multicolumn{7}{|l|}{ In the roles of life (societal): } \\
\hline $\begin{array}{l}\text { Limitations in performing } \\
\text { work or other daily } \\
\text { activities (6) }\end{array}$ & $0.42 \pm 0.41$ & $0.22 \pm 0.35$ & 0.0001 & $0.78 \pm 0.32$ & $0.19 \pm 0.25$ & 0.0001 \\
\hline $\begin{array}{l}\text { Limitations in hobbies or } \\
\text { other pleasures ( } 7)\end{array}$ & $0.35 \pm 0.41$ & $0.17 \pm 0.3$ & 0.0001 & $0.73 \pm 0.24$ & $0.14 \pm 0.23$ & 0.0001 \\
\hline \multicolumn{7}{|l|}{ Cognitive: } \\
\hline $\begin{array}{l}\text { Difficulties in } \\
\text { concentrating (20) }\end{array}$ & $0.59 \pm 0.4$ & $0.4 \pm 0.4$ & 0.0001 & $0.86 \pm 0.27$ & $0.41 \pm 0.28$ & 0.0001 \\
\hline Memorizing (25) & $0.84 \pm 0.2$ & $0.76 \pm 0.24$ & 0.003 & $0.94 \pm 0.17$ & $0.9 \pm 0.15$ & 0.55 \\
\hline \multicolumn{7}{|l|}{ Emotional: } \\
\hline Being uptight (21) & $0.81 \pm 0.27$ & $0.71 \pm 0.26$ & 0.017 & $0.89 \pm 0.16$ & $0.62 \pm 0.3$ & 0.003 \\
\hline Worrying (22) & $0.53 \pm 0.4$ & $0.39 \pm 0.39$ & 0.002 & $0.68 \pm 0.37$ & $0.35 \pm 0.36$ & 0.006 \\
\hline Exasperation (23) & $0.72 \pm 0.29$ & $0.63 \pm 0.32$ & 0.031 & $0.78 \pm 0.24$ & $0.54 \pm 0.37$ & 0.036 \\
\hline Dejection (24) & $0.74 \pm 0.28$ & $0.52 \pm 0.42$ & 0.0001 & $0.84 \pm 0.25$ & $0.49 \pm 0.42$ & 0.008 \\
\hline \multicolumn{7}{|l|}{ Social: } \\
\hline Family life (26) & $0.66 \pm 0.38$ & $0.42 \pm 0.42$ & 0.0001 & $0.92 \pm 0.23$ & $0.46 \pm 0.44$ & 0.0001 \\
\hline Social life (27) & $0.40 \pm 0.42$ & $0.21 \pm 0.37$ & 0.0001 & $0.76 \pm 0.34$ & $0.27 \pm 0.36$ & 0.0001 \\
\hline Financial problems & $0.77 \pm 0.2$ & $0.76 \pm 0.2$ & 0.10 & $0.79 \pm 0.22$ & $0.75 \pm 0.26$ & 0.38 \\
\hline Overall quality of life & $0.52 \pm 0.16$ & $0.40 \pm 0.16$ & 0.0002 & $0.52 \pm 0.16$ & $0.39 \pm 0.11$ & 0.0001 \\
\hline Health & $0.38 \pm 0.27$ & $0.16 \pm 0.25$ & 0.0001 & $0.41 \pm 0.35$ & $0.16 \pm 0.27$ & 0.026 \\
\hline $\begin{array}{l}\text { Self-assessment of quality } \\
\text { of life }\end{array}$ & $0.33 \pm 0.28$ & $0.15 \pm 0.26$ & 0.0001 & $0.35 \pm 0.34$ & $0.17 \pm 0.26$ & 0.047 \\
\hline
\end{tabular}

in work and hobbies occurred in $58.8 \%$ and $65.1 \%$, respectively. During palliative care, $17.5 \%$ and $25.4 \%$ of patients assessed their health and quality of life as very bad. Only $5 \%$ of patients did not experience significant intensity of symptoms. Most of them suffered from several severe symptoms or problems whose intensity was assessed as significant. The most common symptoms experienced by patients as significant were symptoms such as shortness of breath when climbing stairs (84.1\%) and walking (82.5\%), and fatigue (77.7\%).

Intensification of dyspnea at rest, walking up and down the stairs, coughing, constipation, pain, loss of appetite, weakness, tingling hands or feet and difficulty sleeping were observed at 3 weeks after the start of palliative care. In the group of 21 patients who evaluated their quality of life at 6 weeks after the start of palliative care, exacerbation of dyspnea, pain, chest pain, weakness, loss of appetite, constipation, fatigue and shortness of breath at rest and walking up and down the stairs were observed.

In all detailed aspects of functioning about which patients were asked during the study, there was a significant deterioration in the quality of life ( $p<0.0001$ ), except problems with remembering 
Table III. Changes in quality of life in areas of functioning between $1^{\text {st }}$ and $2^{\text {nd }}$ and $1^{\text {st }}$ and $3^{\text {rd }}$ study

\begin{tabular}{|lllcccc|}
\hline Changes in quality of life & & \multicolumn{5}{c|}{ Areas of functioning } \\
\cline { 3 - 7 } & & PH & EM & COG & WEL & SOC \\
\hline Between $1^{\text {st }}$ and $2^{\text {nd }}$ study $(n=63)$ & Average & $-0.11^{*}$ & $-0.14^{*}$ & $-0.13^{*}$ & $-0.21^{*}$ & $-0.19^{*}$ \\
\cline { 2 - 7 } & SD & 0.15 & 0.26 & 0.20 & 0.27 & 0.26 \\
\hline Between $1^{\text {st }}$ and $3^{\text {rd }}$ study $(n=21)$ & Average & $-0.43^{*}$ & $-0.30^{*}$ & $-0.24^{*}$ & $-0.48^{*}$ & $-0.59^{*}$ \\
\cline { 2 - 7 } & SD & 0.27 & 0.28 & 0.25 & 0.38 & 0.35 \\
\hline
\end{tabular}

${ }^{*} p$ < 0.0001; PH - quality of life in physical functioning (questions 1-5 of QLQ-C30 questionnaire); EM - quality of life in emotional functioning (questions 21-24 of QLQ-C30 questionnaire); COG - quality of life in cognitive functioning (questions 20 and 25 of QLQ-C30 questionnaire); WEL - quality of life in welfare functioning (questions 26 and 27 of QLQ-C30 questionnaire); SOC - quality of life in social functioning - social roles (questions 6 and 7 of QLQ-C30 questionnaire).

rated between the first and third assessment and financial problems rated between the first and second as well as the first and third assessment. In the comprehensive assessment, significant deterioration of functioning in all areas compared between the first and second as well as the first and third assessment was observed.

There was a decrease in self-assessment of health and quality of life in all patients as the disease progressed. Among 63 patients in the first assessment, 11 (17.5\%) rated their health as 'very bad', and in the next assessment as many as 40 (63.5\%) patients. Similarly, self-assessment of the quality of life decreased in the first and second assessment. In the first assessment there were $16(25.4 \%)$ patients who rated their quality of life as 'very bad' and as many as 43 (68.3\%) during the second assessment. For 21 patients who survived up to the third assessment, self-assessment of health and quality of life also decreased with time. Only 1 (4.7\%) patient rated her health as 'very bad' in the first assessment, but as many as 14 (66.6\%) in the third assessment. Similarly, self-assessment of the quality of life also decreased in the first and third assessment. In the first assessment 'very bad' was reported by 2 (9.5\%) patients and by 15 $(71.4 \%)$ in the third assessment.

Trajectory of symptoms and physical, emotional, social, societal and cognitive functioning in relation to the time of death (Table IV, Figures 1 and 2)

In order to know the trajectory of symptoms and functioning in various spheres being evaluated by patients in relation to impending death, an analysis was performed for two study groups separately: group 1 included 21 patients who lived over 6 weeks after the start of palliative care and participated in all three assessments; group 2 included 42 patients who lived 3-6 weeks and died before the third assessment.

After the beginning of palliative care (study 1) and in its further course (study 2) a significant difference in the discomfort associated with the feeling of fatigue, pain, loss of appetite, sleep disorders, and constipation between group 1 and group 2 was observed. Patients who lived shorter (group 2) experienced these symptoms as worse. This difference became apparent also in the case of dyspnea in study 2 as a result of deterioration of the feeling of breathlessness in patients in the last days of their lives. In the last weeks before death fatigue was the strongest symptom reducing quality of life according to the opinion presented by patients. In patients with a shorter survival time (group 2) it was already judged very bad during the initial period (average: $0.07 \pm 0.13$ ), while in group 1 it deteriorated significantly, reaching very low values in the last days of life (mean: $0.06 \pm 0.11$ ). Alongside fatigue there were two other symptoms experienced as worst by patients and intensifying until the time of death: constipation and dyspnea.

Functioning in all spheres that had an effect on the assessment of the quality of life by patients was worse in patients with a shorter survival time (group 2) already during the initial period. It deteriorated within a few weeks, reaching very low values in the patient's self-assessment especially in the physical, societal and social areas. In patients with a slightly longer survival (group 1), deterioration in functioning in all spheres was observed during the last weeks of life (between the second and third assessment).

\section{Discussion}

In the current study, the average time of palliative care was only 4 weeks. This indicates the application of the sequential model in practice, in which palliative care is introduced after the causal treatment is finished, when it has failed and/or when it has already been abandoned. It happens despite the good availability of palliative care, especially for cancer patients, and promotion of a model of coexistence of anticancer treatment with palliative care. However, it has recently been 
Table IV. Trajectories of the quality of life depending on the symptoms and functioning

\begin{tabular}{|c|c|c|c|c|c|c|c|}
\hline & Study & Specification & & & e of palliative & & \\
\hline & & & $\leq 7 \mathrm{da}$ & & 3-4 we & & 6-7 weeks \\
\hline & & & Average \pm SD & $P$-value & Average \pm SD & $P$-value & Average \pm SD \\
\hline & 1 & Fatigue & $0.49 \pm 0.25$ & $<0.001$ & $0.36 \pm 0.27$ & $<0.001$ & $0.06 \pm 0.11$ \\
\hline & 2 & Fatigue & $0.07 \pm 0.13$ & & $0.04 \pm 0.07$ & & \\
\hline & 1 & Nausea and vomiting & $0.94 \pm 0.10$ & 0.287 & $0.98 \pm 0.06$ & 0.545 & $0.98 \pm 0.05$ \\
\hline & 2 & Nausea and vomiting & $0.97 \pm 0.08$ & & $0.99 \pm 0.04$ & & \\
\hline & 1 & Pain & $0.67 \pm 0.22$ & $<0.001$ & $0.55 \pm 0.22$ & 0.026 & $0.42 \pm 0.21$ \\
\hline & 2 & Pain & $0.42 \pm 0.18$ & & $0.41 \pm 0.21$ & & \\
\hline & 1 & Lack of appetite & $0.84 \pm 0.23$ & $<0.001$ & $0.70 \pm 0.31$ & $<0.001$ & $0.56 \pm 0.27$ \\
\hline है & 2 & Lack of appetite & $0.49 \pm 0.28$ & & $0.35 \pm 0.30$ & & \\
\hline है & 1 & Dyspnea & $0.65 \pm 0.27$ & 0.078 & $0.51 \pm 0.23$ & $<0.001$ & $0.30 \pm 0.21$ \\
\hline & 2 & Dyspnea & $0.52 \pm 0.25$ & & $0.20 \pm 0.21$ & & \\
\hline & 1 & Sleep disturbances & $0.79 \pm 0.17$ & 0.029 & $0.71 \pm 0.32$ & 0.001 & $0.65 \pm 0.31$ \\
\hline & 2 & Sleep disturbances & $0.62 \pm 0.27$ & & $0.37 \pm 0.37$ & & \\
\hline & 1 & Constipation & $0.76 \pm 0.34$ & $<0.001$ & $0.46 \pm 0.41$ & $<0.001$ & $0.27 \pm 0.36$ \\
\hline & 2 & Constipation & $0.28 \pm 0.36$ & & $0.06 \pm 0.15$ & & \\
\hline & 1 & Diarrhea & $0.95 \pm 0.12$ & 0.545 & $1.00 \pm 0.00$ & 0.994 & $1.00 \pm 0.00$ \\
\hline & 2 & Diarrhea & $0.98 \pm 0.07$ & & $1.00 \pm 0.00$ & & \\
\hline & 1 & Physical & $0.52 \pm 0.29$ & $<0.001$ & $0.41 \pm 0.28$ & $<0.001$ & $0.09 \pm 0.9$ \\
\hline & 2 & Physical & $0.12 \pm 0.16$ & & $0.02 \pm 0.06$ & & \\
\hline & 1 & Performing social roles & $0.75 \pm 0.33$ & $<0.001$ & $0.52 \pm 0.35$ & $<0.001$ & $0.17 \pm 0.22$ \\
\hline 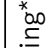 & 2 & Performing social roles & $0.20 \pm 0.30$ & & $0.03 \pm 0.12$ & & \\
\hline 高 & 1 & Emotional & $0.80 \pm 0.19$ & 0.008 & $0.64 \pm 0.30$ & 0.075 & $0.50 \pm 0.29$ \\
\hline$\underbrace{\stackrel{5}{2}}_{4}$ & 2 & Emotional & $0.65 \pm 0.22$ & & $0.52 \pm 0.27$ & & \\
\hline 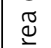 & 1 & Cognitive functioning & $0.90 \pm 0.19$ & $<0.001$ & $0.88 \pm 0.18$ & $<0.001$ & $0.66 \pm 0.20$ \\
\hline & 2 & Cognitive functioning & $0.62 \pm 0.26$ & & $0.42 \pm 0.18$ & & \\
\hline & 1 & Social & $0.84 \pm 0.26$ & $<0.001$ & $0.69 \pm 0.36$ & $<0.001$ & $0.37 \pm 0.37$ \\
\hline & 2 & Social & $0.38 \pm 0.33$ & & $0.13 \pm 0.19$ & & \\
\hline
\end{tabular}

${ }^{*}$ Assessment of quality of life based on questions from QLQ-C3O and QLQ-LC13 questionnaires in case of: fatigue (questions 10, 12, 18), nausea and vomiting (questions 14,15), pain (questions 9, 19), loss of appetite (question 13), dyspnea (question 8), difficulty sleeping (question 11), constipation (question 16), diarrhea (question 17). *Assessment of quality of life based on questions from QLQ-C30 questionnaire in case of: physical functioning (questions 1-5), emotional (questions 21-24), cognitive - memory and concentration (questions 20 and 25), social (questions 26 and 27), performing social roles (questions 6 and 7). Group 1 - 21 patients, who lived 6-8 weeks from the start of palliative care and participated in all three studies; group 2-42 patients, who lived up to 6 weeks from the start of palliative care and participated only in two studies.

pointed out that the model of coexistence of oncology and palliative care is not sufficiently effective in alleviating the symptoms and responding to the needs of patients and their families $[12,16]$.

Oncologists often have concerns that offering palliative care during cancer treatment may indicate that they do not believe in the efficacy of anticancer treatment, since it involves reaching for help from professionals who are associated with impending death. A survey performed among medical oncologists and midlevel providers in the United States showed that palliative care was synonymous with care at the end of life for more than half of them [17]. The term 'palliative care' itself is considered by many of the respondents as a barrier to referral of patients to consult a spe- 


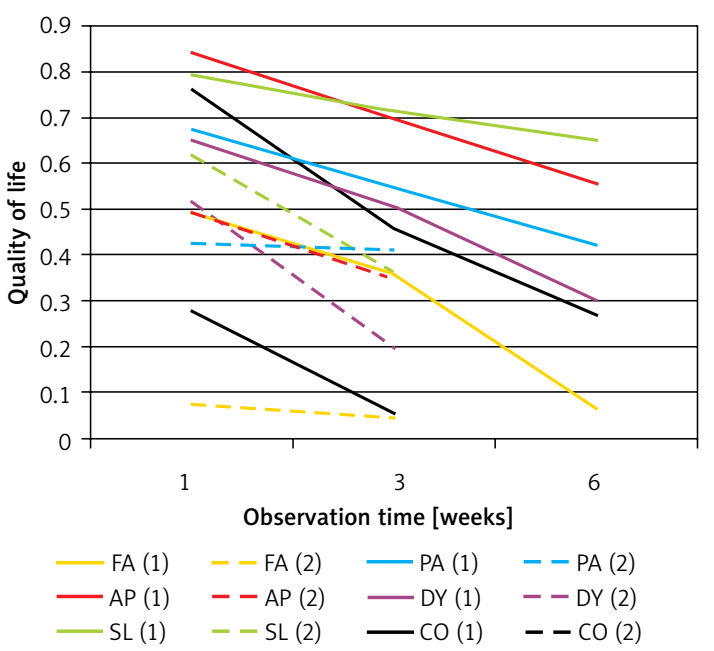

Figure 1 . Trajectory related to quality of life symptoms during the last weeks of life

FA - fatigue assessed by patients based on questions no. 10, 12, 18 from QLQ-C30 and QLQ-LC13 questionnaires, $P A-$ pain (questions 9,19), AP - loss of appetite (question 13), $D Y$ - dyspnea (question 8), SL - difficulty sleeping (question 11), CO - constipation (question 16). (1) groups 1 to 21 , patients who lived 6-8 weeks from the start of palliative care and participated in all three studies; (2) - groups 2 to 42, patients who lived up to 6 weeks from the start of palliative care and participated only in two studies.

cialist, a reason to cause distress or to weaken hope. Stating 'patient referral to palliative care' is still one of the most difficult challenges for oncologists in the field of clinical communication. It is hoped that the situation will improve the message coming from studies evaluating early integration of palliative care with oncology, indicating the effectiveness of such procedures not only in alleviating the physical and psychological symptoms but also in achieving longer survival of patients and reducing the burden on caregivers [6, 18-21]. Interestingly, it was also shown that patients undergoing palliative care had greater satisfaction with their treatment [22]. Taking into account all of these observations, ASCO defines palliative care as that which is 'focused on the relief of suffering, in all of its dimensions, throughout the course of a patient's illness', and the National Cancer Institute emphasizes that: 'Palliative care is given throughout a patient's experience with cancer' $[11,23]$. The present study indicates that there is still no real integration of oncologists' activities and palliative care specialists, which would lead to the early commencement of palliative care in patient with advanced lung cancer.

The importance of even such a short time that is left to the patients with respect to the quality of life dependent on symptoms and functioning in various areas of life is another message from the present study. In short, this relationship could be called as 'the later, the harder'. It was highlight-

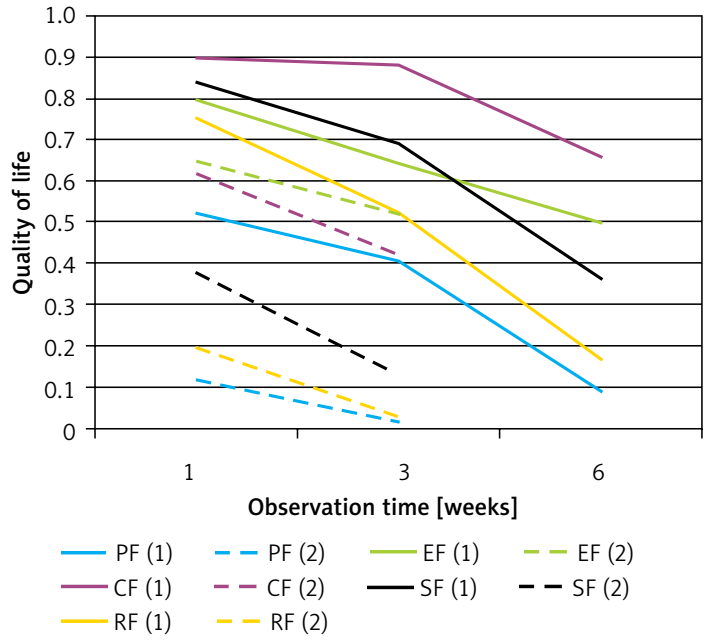

Figure 2. Trajectory related to quality of life depending on functioning areas during the last weeks of life

PF - physical functioning assessed by patients on the basis of questions 1-5 from QLQ-C30 questionnaire, $E F$ - emotional functioning (questions 21-24), CF cognitive functioning - memory and concentration (questions 20 and 25), SF - social functioning (questions 26 and 27), RF-performing social roles (questions 6 and 7). Groups of patients as in Figure 1.

ed by comparative analysis of the two groups of patients including those defined by the length of survival. Patients undergoing palliative care at the time of their last 3-6 weeks of life felt more discomfort associated with symptoms and limitations of functioning in all surveyed areas compared with patients who had been referred to palliative care a few weeks earlier. It could be argued that the later the patient was taken to palliative care, the smaller were the benefits. We should be aware that the period of moving towards death is characterized by progressive weakness and a number of limitations in various spheres of functioning. However, there are a number of problems possible to reverse, control or mitigate, such as dyspnea, constipation and other symptoms as well as the emotional or social needs.

In the present study a gradual deterioration in the quality of life also associated with those needs was observed. Fatigue, constipation and dyspnea were among the worst symptoms endured by patients. Other researchers comparing the quality of life in patients in the palliative medicine ward or receiving palliative care at home also observed significant intensification of symptoms, dyspnea, pain, fatigue, constipation, and loss of appetite in particular [15]. They did not observe improvement in their mitigation in most cases. In the present study dyspnea deteriorated considerably during the last days of life. This observation is consistent with the conclusions of a study of nearly 6000 patients with malignant and non-malignant diseases, who received palliative care, showing that in 
patients with lung cancer the frequency and intensity of dyspnea increased dramatically in the last days of life [24]. Undoubtedly, shortness of breath at the end of life is still a challenge for specialists in palliative medicine. But this raises the question about the relationship between the perception of dyspnea in the last days of life and how it was treated in an earlier period. Interesting observations are associated with the last assessed model to provide care for patients suffering from dyspnea in the course of advanced disease with poor prognosis called Breathlessness Intervention Service (BIS). The BIS is a complex multi-disciplinary intervention using evidence-based non-pharmacological and pharmacological interventions to support patients with advanced disease. It has been shown that the use of 2 weeks of BIS intervention reduced fear and anxiety, and increased confidence in managing breathlessness [25].

In the present study constipation was another symptom, alongside dyspnea, badly tolerated by patients. In patients with a shorter survival time this symptom was very badly assessed at the beginning, becoming worse in the course of palliative care. However, in patients with a slightly longer survival time, constipation was tolerated worse as the duration of palliative care increased, obtaining low values in the last days of life. This may be surprising since the implementation of appropriate prophylactic and/or therapeutic treatment should allow satisfactory alleviation of the symptom in most cases. The present study suggests that patients have not benefited from an effective method of preventing and/or treating this symptom before taking palliative care. Moreover, it was found that palliative care (especially in group 2) did not improve the effectiveness in relieving constipation.

According to the patients' opinion, fatigue was the symptom most strongly related to deterioration of the quality of life. In patients with a shorter survival rate (group 2) fatigue was assessed as a worst symptom already at the beginning of the study, while in group 1 it deteriorated significantly, reaching very low values in the last days of life. Appreciating the prevalence and impact of this symptom on quality of life, the National Comprehensive Cancer Network recommends screening for fatigue in each patient with cancer during the first visit and then at regular intervals and in situations requiring such conduct [26]. In the present study, late initiation of palliative care in patients suffering from extreme fatigue during the last weeks of life could be associated with limited possibilities of its mitigation. Undoubtedly, it had an impact on another parameter, namely quality of life, comprehensively assessed by patients, which worsened continuously and in the last days/weeks before death was defined as 'very bad' by more than $2 / 3$ of patients.

In conclusion, the present study shows that recommendations for early initiation and/or integration of oncology with palliative care are not applied in practice and patients with advanced lung cancer receive palliative care only in the last weeks of life. It seems, however, that even within this short period of time the implementation of palliative care is still important for the quality of life that is assessed by the patient. Patients with a survival time shorter than 6 weeks tolerated symptoms and their functioning worse throughout the palliative care until their death compared with patients with slightly longer survival (6-8 weeks). Fatigue, constipation and dyspnea are among the worst endured symptoms, and the latter intensified in the last period before death. The functioning of the patients deteriorated throughout the whole period of palliative care, especially in the physical, social and societal spheres of life. Late initiation of palliative care for patients with lung cancer may result in limited effectiveness in improving the quality of life, depending on symptoms experienced by them and their ability to function in the physical, emotional, cognitive, social and societal spheres. The principles of early integration of oncology with palliative care should be implemented in Poland, especially in the case of patients with lung cancer, for whom the numerous problems and discomfort associated with symptoms experienced by patients are the highest among cancer patients.

\section{Conflict of interest}

The authors declare no conflict of interest.

\section{References}

1. Ramalingam SS, Owonikoko TK, Khuri FR. Lung cancer: new biological insights and recent therapeutic advances. Cancer J Clin 2011; 61: 91-112.

2. Wojciechowska U. Didkowska J. Nowotwory w Polsce w 2012 roku. Nowotwory J Oncol 2013; 3: 197-216.

3. Cooley ME. Symptoms in adults with lung cancer. A systematic research review. J Pain Symptom Manage 2000; 19: 137-53.

4. McCarthy EP, Phillips RS, Zhong Z, Drews RE, Lynn J. Dying with cancer: patients' function, symptoms, and care preferences as death approaches. J Am Geriatr Soc 2000; 48 (5 Suppl.): 110-21.

5. Kendall M, Carduff E, Lloyd A, et al. Different experiences and goals in different advanced diseases: comparing serial interviews with patients with cancer, organ failure, or frailty and their family and professional care. J Pain Symptom Manage 2015; 50: 216-24.

6. Temel JS, Greer JA, Muzikansky A, et al. Early palliative care for patients with metastatic non-small cell lung cancer. N Engl J Med 2010; 363: 733-42.

7. Dahlin CM, Kelley JM, Jackson VA, Temel JS. Early palliative care for lung cancer: improving quality of life and increasing survival. Int J Palliat Nurs 2010; 16: 420-3. 
8. Irwin KE, Greer JA, Khatib J, Temel JS, Pirl WF. Early palliative care and metastatic non-small cell lung cancer: potential mechanisms of prolonged survival. Chron Respir Dis 2013; 10: 35-47.

9. Temel JS, Greer JA, Admane S, et al. Longitudinal perceptions of prognosis and goals of therapy in patients with metastatic non-small-cell lung cancer: results of a randomized study of early palliative care. J Clin Oncol 201; 129: 2319-26.

10. Johnston B, Buchanan D, Papadopoulou C, Sandeman $\mathrm{G}$, Lord $\mathrm{H}$. Integrating palliative care in lung cancer: an early feasibility study. Int J Palliat Nurs 2013; 19: 433-7.

11. Smith TJ, Temin S, Alesi ER, et al. American Society of Clinical Oncology provisional clinical opinion: the integration of palliative care into standard oncology care. J Clin Oncol 2012; 30: 880-7.

12. Roenn JH, Voltz R, Serrie A. Barriers and approaches to the successful integration of palliative care and oncology practice. J Natl Compr Canc Netw 2013; 11: 11-6.

13. Marchetti P, Voltz R, Rubio C, et al. Provision of palliative care and pain management services for oncology patients. J Natl Compr Canc Netw 2013; 11: 17-27.

14. Hannon B, Swami N, Pope A, et al. The oncology palliative care clinic at the Princess Margaret Cancer Centre an early intervention model for patients with advanced cancer. Support Care Cancer 2015; 23: 1073-80.

15. Leppert W, Turska A, Majkowicz M, Dziegielewska S, Pankiewicz P, Mess E. Quality of life in patients with advanced lung cancer treated at home and at a palliative care unit. Am J Hosp Palliat Care 2012; 29: 379-87.

16. Bruera E, Hui D. Conceptual models for integrating palliative care at cancer centers. J Palliat Med 2012; 5: 1261-9.

17. Fadul N, Elsayem A, Palmer IL, et al. Supportive versus palliative care: what's in a name? A survey of medical oncologists and midlevel providers at a comprehensive cancer center. Cancer 2009; 115: 2013-21.

18. Pirl WF, Greer JA, Traeger L, et al. Depression and survival in metastatic non-small-cell lung cancer: effects of early palliative care. J Clin Oncol 2012; 30: 1310-5.

19. Bandieri E, Sichetti D, Romero M, et al. Impact of early access to a palliative/supportive care intervention on pain management in patients with cancer. Ann Oncol 2012; 23: 2016-20.

20. Bakitas MA, Tosteson TD, Lyons KD, et al. Early versus delayed initiation of concurrent palliative oncology care: patient outcomes in the ENABLE III Randomized Controlled Trial. J Clin Oncol 2015; 33: 1438-45.

21. Dionne-Odom JN, Azuero A, Lyons KD, et al. Benefits of early versus delayed palliative care to informal family caregivers of patients with advanced cancer: outcomes from the ENABLE III Randomized Controlled Trial. J Clin Oncol 2015; 33: 1446-52.

22. Zimmermann C, Swami N, Krzyzanowska M, et al. Early palliative care for patients with advanced cancer: a cluster-randomized controlled trial. Lancet 2014; 383: 1721-30.

23. The National Cancer Institute ( $\mathrm{NCl})$ fact sheet on palliative care. Available at: http://www.cancer.gov/aboutcancer/advanced-cancer/care-choices/palliative-carefact-sheet\#q1.

24. Currow DC, Smith J, Davidson PM, Newton PJ, Agar MR, Abernethy AP. Do the trajectories of dyspnea differ in prevalence and intensity by diagnosis at the end of life? A consecutive cohort study. J Pain Symptom Manage 2010; 39: 680-90.
25. Farquhar MC, Prevost AT, McCrone P, et al. Is a specialist breathlessness service more effective and cost-effective for patients with advanced cancer and their carers than standard care? Findings of a mixed-method randomized controlled trial. BMC Med 2014; 12: 194.

26. Mock V, Atkinson A, Barsevick A, et al. National Comprehensive Cancer Network. NCCN Practice Guidelines for Cancer-Related Fatigue. Oncology 2000; 14: 151-61. 